\title{
Burst increases of precipitating electrons recorded by the DEMETER satellite before strong earthquakes
}

\author{
X. Zhang ${ }^{1,2}$, C. Fidani ${ }^{3}$, J. Huang ${ }^{1}$, X. Shen ${ }^{1}$, Z. Zeren ${ }^{1}$, and J. Qian ${ }^{1}$ \\ ${ }^{1}$ Institute of Earthquake Science, China Earthquake Administration, Beijing, 100036, China \\ ${ }^{2}$ INFN Sezione di Perugia, Via A. Pascoli, Perugia, 06123, Italy \\ ${ }^{3}$ Central Italy Electromagnetic Network, Via Fosso del Passo 6, 63900 Fermo, Italy
}

Correspondence to: X. Zhang (zhangxm96@hotmail.com)

Received: 23 July 2012 - Revised: 27 November 2012 - Accepted: 12 December 2012 - Published: 29 January 2013

\begin{abstract}
This case study developed a method for data processing over six years, from 2004 to 2010, of $70 \mathrm{keV}-$ 2.3 MeV electrons recorded by the DEMETER (Detection of Electro-Magnetic Emissions Transmitted from Earthquake Regions) satellite. Short time increases in electron counting rates, having $99 \%$ probabilities of not being Poisson fluctuations, were statistically selected using geomagnetic invariant space and called electron bursts. Temporal series were analysed confirming the seasonal variations in low energy bands of 70-450 keV. Differently from previous results, the DEMETER results exhibited two peaks of electron bursts: one in the period June-August and one in the period DecemberFebruary annually. Specifically, six earthquake cases are presented in detail having increases in electron burst number prior to events. Moreover, electron burst precipitation occurring before each strong earthquake of the entire period over the life of the satellite with $M \geq 7.0$ was verified as having a probability greater than $97 \%$ of not being of a statistical origin. Low energetic electrons in $70-330 \mathrm{keV}$ resulted occurring more frequently near seismic activity than those observed in $330 \mathrm{keV}-2.34 \mathrm{MeV}$ energy bands at the satellite altitude in the ionosphere.
\end{abstract}

\section{Introduction}

Over the past decade, correlations between high energy electron bursts (EBs) in near Earth space and earthquakes have been reported in several studies. Sharp, short-term increases in particle counting rates (CRs), ranging from tens of seconds to a few minutes, were called EBs by Voronov et al. (1987). Results from a study by Aleksandrin et al. (2003), based on various space experiments including the Mir orbital station, Meteor-3, Gamma and SAMPEX (Solar Anomalous and Magnetospheric Particle Explorer) satellites, observed EBs several hours before strong earthquakes. Also, Sgrigna et al. (2005) carried out an investigation on data recorded by the SAMPEX satellite, reporting the existence of a shortterm seismic precursor of $\sim 4 \mathrm{~h}$ in high energy electrons $(E \geq$ $4 \mathrm{MeV})$. At lower energy, Boskova et al. (1994) observed enhanced electron fluxes onboard the Intercosmos 24 satellite before the 1990 Iranian earthquake. Fidani and Battiston (2008) analysed data over $10 \mathrm{yr}$ recorded by the NOAA15 satellite, where electron energy was found to be lower than $100 \mathrm{keV}$. The corresponding EBs were observed near the boundary of the South Atlantic Anomaly (SAA) prior to strong earthquakes with magnitudes larger than 6.5 in the Indonesian region.

A possible model for describing high energy particle precipitation associated with seismic activity might include ultra-low frequency (ULF) and very low frequency (VLF) electromagnetic emissions (EME), which have been reported to be generated in seismic regions and are believed to propagate upwards into the atmosphere, ionosphere and even into the magnetosphere (Molchanov and Majaeva, 1994; Pulinets and Boyarchuk, 2004; Sidiropoulos et al., 2011). In fact, the magnetosphere is characterised by regions that are filled with charged particles trapped in the geomagnetic field, known as Van Allen belts (VAB) (McIlwain, 1966). These particles are trapped only for well-defined values of the angles between their velocity and the geomagnetic field, known as pitch angles. If the EME frequencies are near the characteristic motions of charged particles in VAB, an energy exchange can occur changing the particle pitch angles, and 
thereby inducing their exit from the trapping condition (Abel and Thorne, 1998). To this regard, Imhof et al. (1981a) report on narrow peaks in energy spectra of inner radiation belt electrons. Other case studies (Datlowe et al., 1985) have suggested that electron accelerations are due to geomagnetic fluctuations that have periods comparable with azimuthal drift periods of electrons. The same authors compared these energy spectra with those of precipitating electrons from the inner VAB due to VLF transmitters (Imhof et al., 1981b), describing differences (Datlowe, 2006). Precipitation phenomena are made up of particle absorptions in the atmosphere, occurring when the bouncing points of the particles fall into low altitudes $(<100 \mathrm{~km})$, due to pitch angle perturbations. Along the bouncing motions, particles drift around the Earth along their equivalent field lines, which can be quantified by the parameter $L$ indicating the set of equivalent lines around the globe. Given this, these particles can be detected in all the regions under the VAB, when the satellites cross through different L-shell regions (Galper et al., 1997).

Since the launch of the DEMETER (Detection of ElectroMagnetic Emissions Transmitted from Earthquake Regions) satellite on 29 June 2004, several studies investigating high energy particle precipitation have been published. To this regard, Parrot et al. (2006) reported on an earthquake having a magnitude of 7.3, and taking place on 22 November 2004, northeast of New Zealand $\left(46.57^{\circ} \mathrm{S}, 164.83^{\circ} \mathrm{N}\right)$. Just over the epicenter area of this earthquake, increases in the particle fluxes at the two closest orbits in local nighttime were observed. Immediately after this, an electrostatic turbulence was recorded. Additionally, a decrease in the particle flux over this seismic region, accompanied by electrostatic turbulence, was observed during the next orbit passing over the epicenter area during the daytime. Furthermore, Sauvaud et al. (2008) investigated for a relationship between energetic electrons and ground-based VLF transmitter signals and reported enhancements in the $\sim 100-600 \mathrm{keV}$ drift-loss cone electron fluxes at $L$ values between 1.4 and 1.7 being due to the Australian NWC operations and ionospheric absorption. Gemelos et al. (2009) reported that seasonal variations in energetic electrons were consistent with lighting-induced electron precipitation (LEP) over North America. These energetic electron fluxes in the slot region (between $L=2$ and 3 ) were significantly higher in the summer than in the winter because of significant lightning activity in the Northern Hemisphere. In a recent work (Buzzi, 2008), there were no observed significant correlations between EBs and seismic events in DEMETER Instrument for the Detection of Particle (IDP) data. However, the EB behaviour was considered for only $18 \mathrm{~h}$ prior to earthquakes. Being so, further research must be also carried out on a wider time scale to confirm such a result.

In this study here, the high energy particle flux data detected by IDP on the DEMETER satellite, from 2004 to 2010, were processed by a statistical analysis method and are presented in Sect. 2. A study of EB time evolutions can be found in Sect. 3, while a comparison between EB statistical distributions and seismic activity is reported in Sect. 4, whereas Sect. 5 presents the conclusions.

\section{The IDP database and the data processing method}

The IDP spectrometer on DEMETER was designed to detect for weak electron fluxes at low-latitude, which are principally located below the VAB at the altitude of about $710 \mathrm{~km}$ (Sauvaud et al., 2006). In order to do so, the maximum geometry factor of IDP was defined as $1.2 \mathrm{~cm}^{2} \mathrm{sr}$. The calculation of the geometry of IDP, as well as its shielding thickness, was performed for the complete energy range (from $70 \mathrm{keV}$ to $2.34 \mathrm{MeV}$ ) using the GEANT-3 code from CERN. The sensor head was located on top of a case that contained the associated analog electronics. The detector was pointed perpendicular to the orbital plane of the satellite, which was almost polar and circular. The pitch angles of the detected particles were therefore close to $90^{\circ}$ (Sauvaud et al., 2006).

The Hbook fortran package developed by CERN for histogram analysis (Couet and Goossens, 1998) was applied to the entire database in an interactive way. Root framework (Brun, 2009) aided in visualising peculiar characteristics of the database or a part of it. Moreover, this framework was used to choose the relevant quantities of multidimensional histograms created by Hbook for analysing electron precipitation. The first step in the preparation of the DEMETER IDP data was to transform all of the IDP binary files into Ntuples data for Hbook by uniforming them into a single time step, chosen to be $4 \mathrm{~s}$. Additionally, the geomagnetic Ap index relative to the same period was introduced. This was done to identify the corresponding geomagnetic activity level in the magnetosphere and the ionosphere. These index data were downloaded from the Global Geomagnetic Activity of GeoForschungsZentrum (GFZ) Potsdam website at ftp://ftp.gfz-potsdam.de/pub/home/obs/kp-ap/tab/. Furthermore, the magnetic filed mirror point, the pitch angle at the Equator, the third adiabatic invariant and other parameters calculated by means of the UNILIB libraries (Krunglanski, 2002) were introduced to better determine the dynamics of the energetic electrons.

The total deposited energy range on the DEMETER ID was divided into 255 channels, and another channel was used to encode all the electron counts corresponding to an energy loss greater than $2.34 \mathrm{MeV}$. IDP can function in two modes: burst and survey (Sauvaud et al., 2006). They are accurately dependent on the geographical satellite positions, so to obtain more information on seismic regions. Under the burst mode, the electron energy spectrum was obtained every second with 256 channels, whereas, in the survey mode, the electron energy spectrum was obtained every $4 \mathrm{~s}$ with 128 channels. The electron flux intensity (unit: elect $\mathrm{cm}^{-2} \mathrm{~s}^{-1} \mathrm{sr}^{-1} \mathrm{KeV}^{-1}$ ) data were stored into different energy channels. However, in analysing the electron flux 

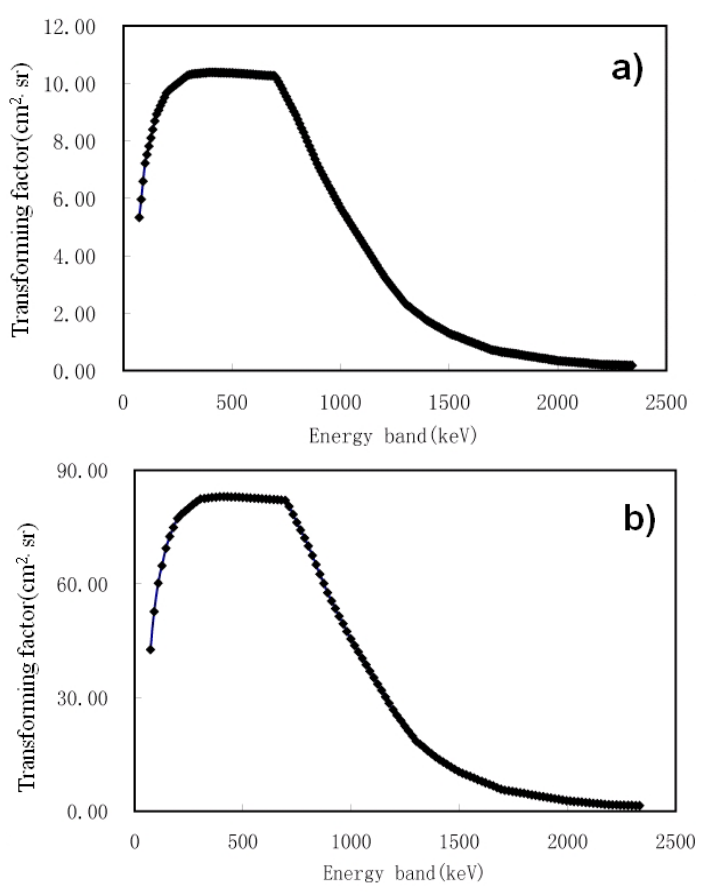

Fig. 1. The transforming factor to convert particle fluxes into CRs, with respect to the electron energy in the burst (a) and survey (b) modes on DEMETER.

in connection with seismic activity, the corresponding CRs during the entire satellite orbit were analysed. To ensure comparability and consistency, the flux data recorded at burst and survey modes were transformed into CR numbers. This was done by multiplying the transforming factor shown in Fig. 1, which was calculated for the different energy channels, and by combining the $1 \mathrm{~s}$ data from the burst mode to obtain the $4 \mathrm{~s}$ data as in the survey mode. Fifteen energy bands were regrouped based upon the 256 and 128 channel data: $72.9-81.8 \mathrm{keV}$ (e1), 90.7-117.4 keV (e2), 126.3$170.8 \mathrm{keV}$ (e3), $179.7-242.0 \mathrm{keV}$ (e4), $250.9-331.0 \mathrm{keV}$ (e5), 339.9-437.8 keV (e6), 446.7-562.4 keV (e7), 571.3$704.8 \mathrm{keV}$ (e8), 713.7-865.0 keV (e9), 873.9-1043.0 keV (e10), $\quad 1051.9-1238.8 \mathrm{keV} \quad$ (e11), $1247.7-1452.4 \mathrm{keV}$ (e12), 1461.3-1683.8 keV (e13), 1692.7-1933.0 keV (e14), 1941.9-2342.4 keV (e15).

The CR analysis was performed in the invariant space of L-shell and pitch angle $\alpha$, where $L$ and $\alpha$ remain constant parameters of the particle motion for small and slow perturbations of the VAB. Being so, particle dynamics is more stable in this space compared to geographical space. The invariant studied area included L-shells less than 1.6 to exclude high latitude regions, and magnetic field intensities larger than $0.22 \mathrm{Gs}$ to exclude SAA. High latitude regions are characterised by very unstable extraterrestrial influence of CRs (Millan and Thorne, 2007), whereas the SAA region principally concerns high fluxes of trapped particles. However, the $L, \alpha$ space still mixes geographical regions of high
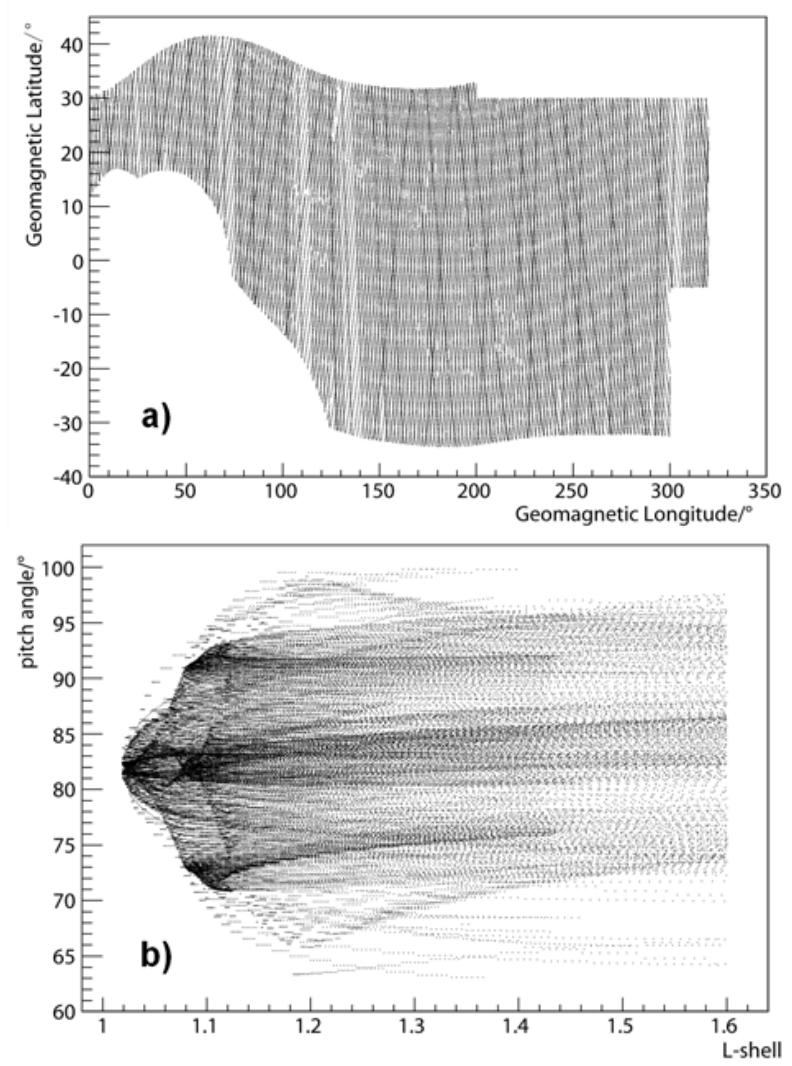

Fig. 2. Studied areas under geomagnetic longitude-latitude coordinates (a) and $L$ shell-pitch angle coordinates (b).

electron fluxes westward of the SAA and weak fluxes over Asia. In order to ensure the reliability of EB selection in connection with terrestrial events, a minimum stable background was sought. The final selected area is shown in both parts of Fig. 2 with respect to the geomagnetic latitudes and longitudes, as well as the invariant coordinate systems. Given the low latitudes of considered CRs, the electron pitch angles were mainly distributed at $60-100^{\circ}$ (Fig. 2b) due to the horizontal orientation of IDP on DEMETER.

A two dimensional matrix $(L, \alpha)$ was used to examine electron CR data and to select CR fluctuations in a statistically significant way. In order to do so, small cells were divided into $L$ bins with a size of 0.01 in the $L$ interval $1.0-1.6$, and $\alpha$ bins with a size of $0.1^{\circ}$ in the $\alpha$ interval 60$100^{\circ}$. To obtain reliable statistics, the satellite was passed through each $(L, \alpha)$ cell at least 20 times. Typical CR distributions at e2 and e8 energy bands inside one small cell are shown in the two distributions of Fig. 3. Here, the CR at the e 2 energy band is much higher than that at the e 8 energy band, which means that more lower energy electrons were trapped at the satellite altitude. The CR distributions are compatible with the Poisson processes as in previous studies (Sgrigna et al., 2005; Fidani et al., 2010). To define the condition for which a CR is not a Poisson fluctuation with $99 \%$ 

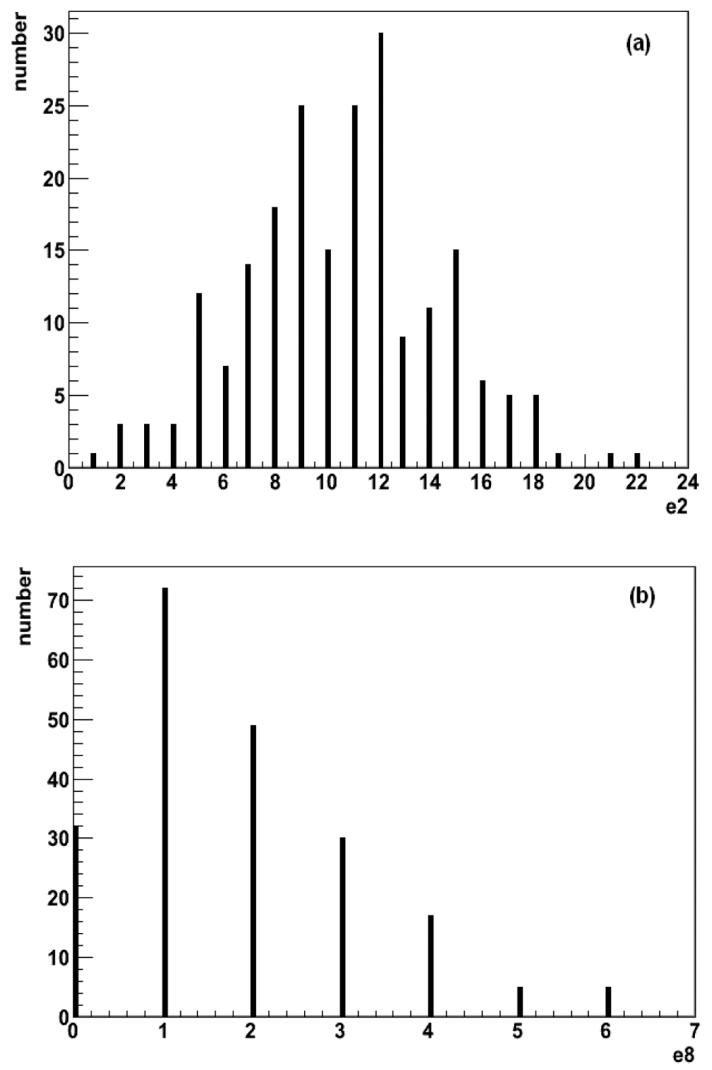

Fig. 3. Typical distribution histograms of 4-s CRs detected over 80 quiet solar days before the considered date, corresponding to the energy bands e 2 (a) and e 8 (b), in the cell with $1.10 \leq L<1.11$ and $92^{\circ} \leq \alpha<92.1^{\circ}$; the number of CR satisfies a Poisson process.

probability, the distribution variance was calculated and the number $n_{\sigma}$ of $\sigma$ was introduced. So, the CR amplitude $x$ has to be greater than the average $\bar{x}$ of $n_{\sigma} \sigma$, which is expressed as $P\left(x>\bar{x}+n_{\sigma} \sigma\right)<0.01 . \sigma=\sqrt{\bar{x}}$ refers to a Poisson distribution, and $\bar{x}$ is the CR average in a well-defined cell. $\bar{x}$ was calculated over the 80 days of quiet solar conditions preceding the day under study, while the quiet solar condition was defined using the Ap index, which was chosen to be less than 20 , as in previous studies (Sgrigna et al., 2005). Since $\bar{x}$ is not usually a small integer number, $n_{\sigma}$ was interpolated using a double exponential fitting (Fidani and Battiston, 2008):

$n_{\sigma}=17.9526-2.7395\left(1-e^{-\bar{x} / 2.8039}\right)-12.0547\left(1-e^{-\bar{x} / 0.3269}\right)$.

$n_{\sigma}$ is large in order to ensure the coverage of a wide CR distribution range whenever the average value is small. Sharp, short-term increases in CR electrons every $4 \mathrm{~s}$ were defined as EBs when the CRs were greater than the $\bar{x}$ of $n_{\sigma} \sigma$.

\section{Analysis of the results}

\subsection{The examination on IDP data}

Due to IDP problems regarding the initial data processing at the DEMETER center, sudden changes between the burst and survey mode data in e1 band occurred, as described by Buzzi (2008). Given this, EBs in the e1 band were excluded from the analysis.

All the energy band electron data were processed to search for the daily EBs using the method described in Sect. 2. The EB time series detected in the e5 band from April 2008 to December 2009 is reported in Fig. 4. Here, there are peaks every 30 days and it was verified that EBs had their highest peaks every 30 days in all of the other energy bands. The number of EBs in Fig. 4 increased to more than 100 monthly, and these EB peaks lasted 5-8 days between April 2008 and March 2009. Figure $4 \mathrm{~b}$ shows that the pitch angle had regular time variations until March 2009. This was due to a problem onboard the satellite with the stellar sensor during periods of full moon (M. Parrot, personal communication, 2010). During these periods the satellite was slightly tilted, but after March 2009 the onboard system was able to take into account this difficulty. Thus, the data relative to days during the full moon periods were not considered before March 2009. Consequently, the pitch angle intervals in Fig. $4 \mathrm{~b}$ ranged between $72^{\circ}$ and $77^{\circ}$ and between $90^{\circ}$ and $92^{\circ}$, resulting relative to precipitating electrons. Furthermore, the number of daily EBs selected with $99 \%$ probability of not being Poisson fluctuations has increased due to the fact that the average $\mathrm{CR}$ is decreased.

\subsection{Long-term variation features of EBs at different energy bands}

After excluding the full moon periods, as well as the disturbed solar days with Ap larger than 20, the EB selection was applied to a total number of 1423 days, obtaining different EB time series for each energy band. Three groups of EB energy bands were evidenced from the behaviour of time series (see Fig. 5): (i) at e2-e3 bands the number of EBs was much higher compared to the other bands, having more than 100 EBs each day, thus exhibiting a clear seasonal period; peaks occurred between June and August and during the winter months, while valleys appeared in the spring and autumn (see Fig. 5a); (ii) at e4-e6 bands the number of EBs shows a more regular behaviour, and the seasonal period was not more clearly defined and disappeared after mid2008 (see Fig. 5b); (iii) at e7-e15 bands there were fewer recorded EBs, less than 10 daily with no evident periodicity (see Fig. 5c). Within the observed energy band on the DEMETER, the number of CRs and selected EBs both declined with the increases in electron energy. Similar results have been reported by Fidani et al. (2008) where the selected EBs from NOAA satellite time series exhibited peak values 

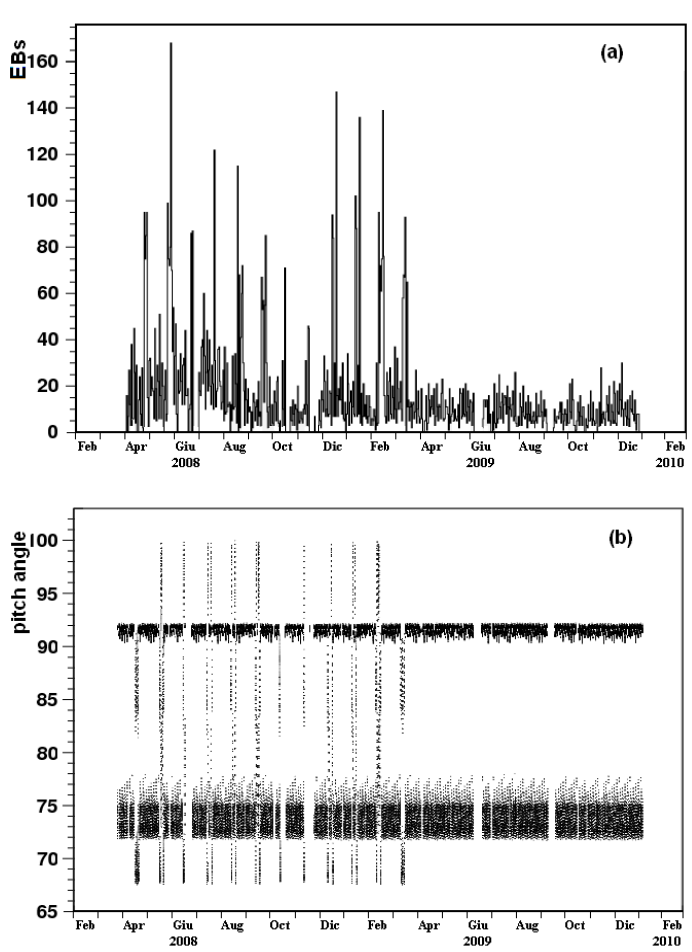

Fig. 4. The number of daily EBs selected with $99 \%$ probability of not being Poisson fluctuations, in the energy band e5 from April 2008 to December 2009 (a); and the pitch angle distribution along the same period in (b). Note that the EB peaks were detected in the geographical region of $200-250^{\circ} \mathrm{E}, 0-20^{\circ} \mathrm{N}$, when particle pitch angles covered the entire range over a few days each month.

between May and August annually, while less markedly in December.

Annual (Vassiliadis et al., 2002) and semiannual (Kanekal et al., 2010) modulations in electron flux were observed in the SAMPEX data, where northern summer and spring with autumn flux increased, respectively. The SAMPEX lowEarth polar orbit at an altitude of about $600 \mathrm{~km}$ and an inclination of $82^{\circ}$ (Baker et al., 1993) was similar to the DEMETER orbit. However, both analyses were developed on larger electron energies and L-shells. Gemelos et al. (2009) also studied the phenomena of lightning-induced electron precipitation and reported annual periodicity in energetic electron fluxes to be significantly higher in the northern summer than in the winter over the United States. The authors compared this finding with the seasonal variations of lightning activity in the Northern Hemisphere. It needs to be noted that the L-shell was in the 2-6 range and the electron energy band was between $2-6 \mathrm{MeV}$ in the three studies cited above, where no restrictions were imposed on electron fluctuation numbers or solar influence. Being so, the semiannual modulation in DEMETER EBs, having two peak numbers occurring in the summer and winter, was analogous to previously published findings. Semiannual modulation characterises the low
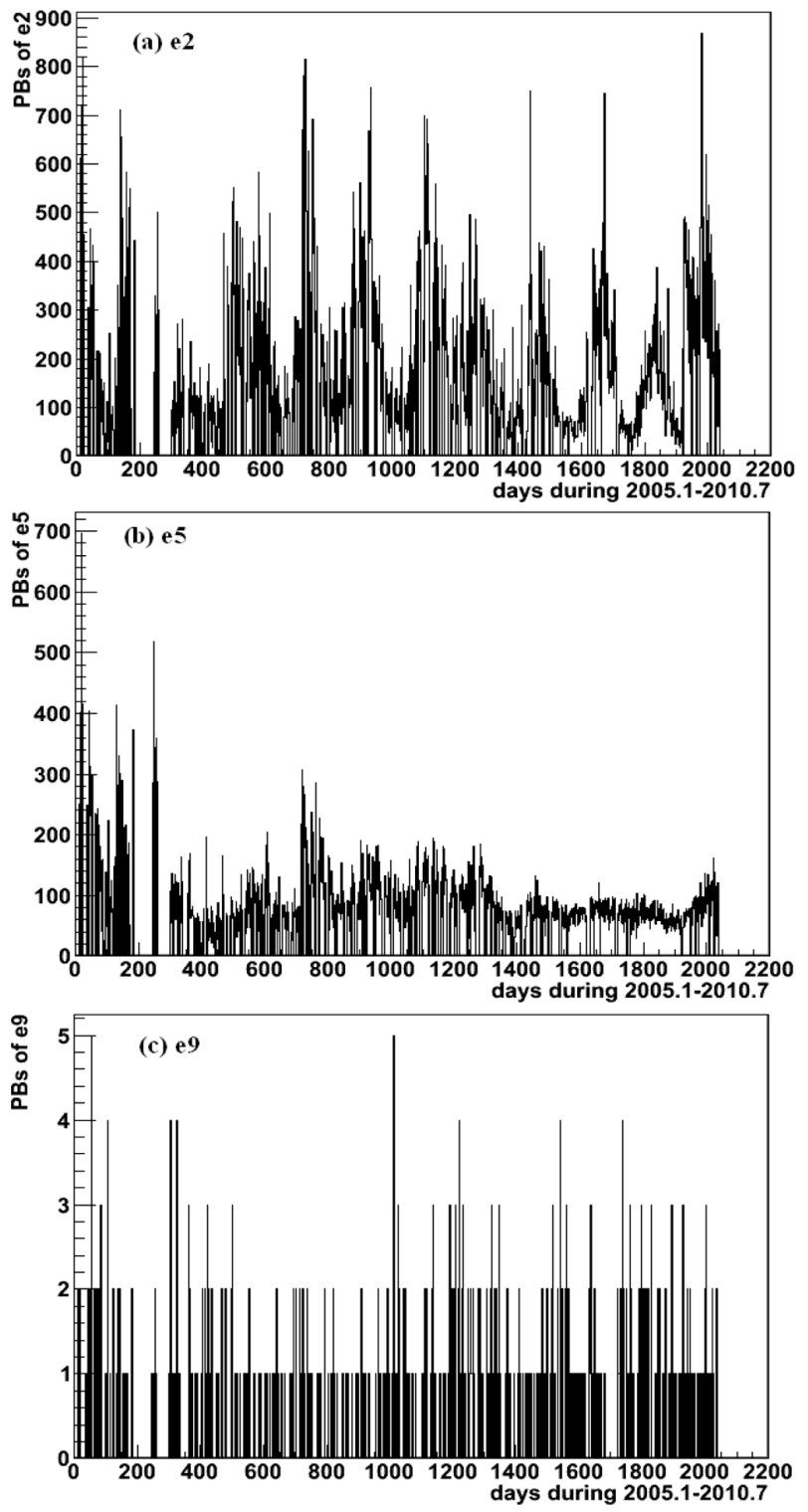

Fig. 5. The number of daily EBs selected having $99 \%$ probability of not being Poisson fluctuations, at different energy bands between February 2005 and June 2010, after eliminating the days where pitch angles were found to be unacceptable: (a) e 2 band; (b) e5 band; (c) e9 band.

L-shell and CR fluctuations of the electron formations under the radiation belts (Grigoryan et al., 2008) during solar quiet periods.

\subsection{Brief-term variation features of EBs at different energy bands}

The time resolution considered in this study was $24 \mathrm{~h}$, as the authors were interested in obtaining time differences with seismic events greater than $18 \mathrm{~h}$. Strong fluctuations in EB numbers occurred from one day to another; see Fig. 5a. Here, 
several days exhibit peaks that were characterised by leaps of several hundreds of EBs at lower energy bands (e2-e3). Strong fluctuations in the EB numbers can be also observed in the e4-e6 bands in Fig. 5b, where increases from 0 to about 100 are more evident during longer times and then reduced in number over the following months. This pattern was strictly linked to both the solar cycle and the Ap threshold that was chosen. There were more selected days, and therefore less discarded days during the final years because of the solar minimum, whereas the Ap index indicated strong and frequent solar influence on the first group of years. Being so, the leaps in Fig. 5b could not be considered true fluctuations and thereby had to be discarded in calculating EB averages and variances. In this study, only the EB numbers of these first five energy channels were analysed in relation with strong earthquakes, as the EB numbers in the e7-e15 energy channels were few and did not provide evidence of significant perturbation.

\section{Correlations between EBs and strong earthquakes}

Here, the results from an initial analysis of EBs, in order to determine the principal parameters to verify if a relationship between electron precipitation and earthquakes exists, are provided. To this regard, six histograms obtained by plotting the total number of half day EBs detected along a limited interval of latitude orbits and occurring around the time of strong earthquakes, having magnitudes $M \geq 7.0$, will be discussed. No longitudinal limit was applied to this study, as electrons drift from 10 to $100 \mathrm{~h}$ all over the globe at these energies. Regarding time limits, full moon periods were excluded from the analysis up to March 2009. In order to quickly pick up the EB anomalous signals with seismic activity, the latitudinal difference between each $\mathrm{EB}$ and the considered earthquake was also evaluated. Finally, the whole set of $M \geq 7.0$ earthquakes will be summarised below, along with the selected EBs within the chosen distances of time and position from the seismic events. The seismic catalogue was downloaded from the USGS website at http://earthquake.usgs.gov/earthquakes/ eqarchives/epic/epic_global.php, providing dates, times, longitudes, latitudes, depths and magnitudes.

\subsection{The anomalous EB increase around the 2005 Sumatra earthquake}

On 28 March 2005, an earthquake with magnitude of 8.6 took place in Sumatra, Indonesia, at $2.09^{\circ} \mathrm{N}, 97.11^{\circ} \mathrm{E}$. This was the biggest aftershock of the 26 December 2004 Sumatra earthquake, which had a magnitude of 9.2. EBs were localised within $\pm 5^{\circ}$ latitudes. Sudden increases in EB were detected in the e 2 and e 3 energy bands. The maximum number of EBs in the e3 band occurred on the 81st day of 2005, 6 days before the Sumatra earthquake on day 87 , as shown in Fig. 6a. The histogram collected EBs 20 days prior to and 20 days after this earthquake, with the maximum reaching 21 EBs compared to the average of 7. The EB histogram satisfies a super-Poisson distribution with a sigma of more than 4 counts. Zhang et al. (2010) studied the ionospheric disturbances recorded by the DEMETER satellite before this earthquake, evidencing significant anomalies in electron densities, temperatures and VLF electric fields on 22 and 23 March. EB precipitation during these two days ranged from $-5^{\circ}$ to $20^{\circ}$ in latitude near $100^{\circ}$ in longitude along the satellite orbit, similar to the perturbations shown in Fig. 2 of Zhang et al. (2010). These might have been due to electron precipitation, if the secondary ionization created by these precipitating electrons had been sufficient to produce an appreciable percentage of the ambient D-region conductivity (Cotts et al., 2011).

\subsection{The anomalous EB increase around the 2007 Indonesia earthquake}

On 21 January 2007, an earthquake off Indonesia, having a magnitude of 7.5 , took place at $1.07^{\circ} \mathrm{N}, 126.28^{\circ} \mathrm{E}$. Figure $6 \mathrm{~b}$ shows the EBs in the e4 band within the latitude of $\pm 4^{\circ}$ from the earthquake position. On the 20th day of 2007, the recorded number of EBs was 16, which was the maximum value reported between days 1 and 50 of 2007 . The EB distribution during the same period was super-Poisson, having an average near 5 and a sigma slightly above 4 counts. The EBs increased again on the 4th (23rd March) day, reaching 14 EBs, which had occurred 17 days before the main shock. The principal EB anomaly occurred one day before the Indonesia earthquake, and the selected EBs were observed near the Equator. The EBs exhibited the anomalies in the e4 band, which indicates that the electron energy was in the 250$330 \mathrm{keV}$ range, a factor 3 greater than the previous energy range of e2.

\subsection{The anomalous EB increase around a 2008 Yutian, China, earthquake}

On 20 March 2008, an earthquake with a magnitude of 7.2 took place in Yutian, China, at $35.49^{\circ} \mathrm{N}, 81.47^{\circ} \mathrm{E}$. EBs were selected within a $\pm 5^{\circ}$ latitude difference, and the maximum number of EBs was recorded in the $\mathrm{e} 3$ band on the 75th day of 2008, 5 days before the earthquake on the 80th day (see Fig. 6c). The EB peak reached 80 counts compared to an average of more than 20 counts and a sigma of nearly 15 counts distributed in a super Poisson fashion. This was a stronger perturbation than the previous two cases, as the number of EBs on the 75th day was four sigmas greater than the average calculated period 20 days before and 20 days after the earthquake. The increases in EB numbers in the e4 and e5 bands were also analysed, but their times of occurrence were prior to and later than those in the e 2 and e 3 bands, which occurred eight and one days before the Sumatra Earthquake, 

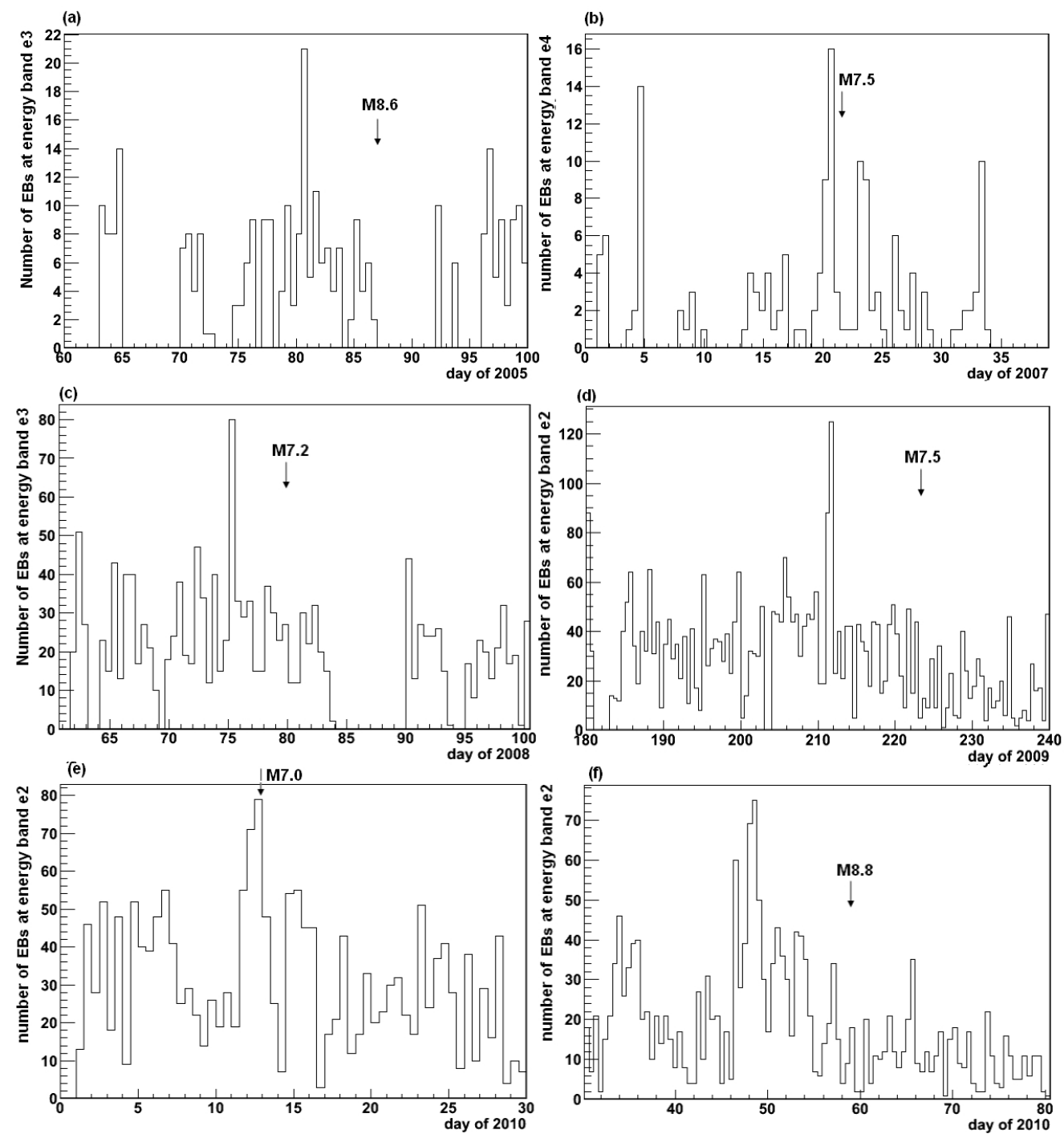

Fig. 6. The number of half-daily EBs in the energy bands of e2, e3 and e4, selected at different latitude intervals around the latitudes of six main shocks greater than 7: (a) Sumatra $M=8.6$ on 28 March 2005; (b) Indonesia $M=7.5$ on 21 January 2007; (c) Yutian $M=7.2$ in China on 20 March 2008; (d) Andaman $M=7.5$ on 10 August 2009; (e) Haiti $M=7.0$ on 12 January 2010; (f) Chile $M=8.8$ on 27 February 2010.

respectively. The significance of the e 4 and e 5 band peaks were lower than the significance of the e 2 and e 3 bands for the Sumatra earthquake.

\subsection{The anomalous EB increase around the 2009 Andaman earthquake}

On 10 August 2009, an earthquake with a magnitude of 7.5 took place off the Andaman Islands, at $14.1^{\circ} \mathrm{N}, 92.9^{\circ} \mathrm{E}$. In this case, EBs were accumulated within a latitude difference of $\pm 5^{\circ}$ compared to the epicentral latitude. The maximum EB number occurred on the 211th day of 2009, 11 days before the earthquake (see Fig. 6d). EB distribution was as with the above three cases super-Poisson and at the highest observed peak in the e 2 channel, where the EB number was
126 with respect to an average of 40 counts and a sigma of more than 27 counts. So, also prior to this earthquake, the e2, e3 and e4 energy bands all exhibited significant increases in EBs. As in the case 4.3, the precipitated electrons were distributed in a wider energy band of $90.7-331.0 \mathrm{keV}$, even if all were observed at the same time before the earthquake.

\subsection{The anomalous EB increase around the 2010 Haiti earthquake}

On 12 January 2010, an earthquake with a magnitude of 7.0 took place in Haiti at $18.44^{\circ} \mathrm{N}, 72.57^{\circ} \mathrm{W}$. Figure 6e shows the EBs in the e2 band in January 2010 at the latitude range of $0-18^{\circ} \mathrm{N}$. It can be seen that the EB number in the e 2 band increased between January 11th and 12th, while on the 13th 
the EB number declined. During January 2010, the EB number reached 80 only on 12 January, more than two sigmas above the average (see Fig. 6e). As in the previously cited cases above, EB distribution was super-Poisson, with an average of 19 and a sigma of more than 5 EB counts. The EBs above latitude $18^{\circ} \mathrm{N}$ did not exhibit any obvious increases, which suggests that EBs occurred prior to and south of the epicenter in the Northern Hemisphere.

\subsection{The anomalous EB increase around the 2010 Chilean earthquake}

On 27 February 2010, an earthquake with a magnitude of 8.8 took place in Chile at $36.12^{\circ} \mathrm{S}, 72.90^{\circ} \mathrm{W}$. Figure of shows the EBs in the e2 band with latitudes above $20^{\circ}$. As this earthquake took place in the SAA region, which was not included in the studied area, the considered EBs were observed exclusively at conjugate points. Before this earthquake, the number of EBs repeatedly vacillated between days 46 and 55, reaching a maximum of $75 \mathrm{~EB}$ counts on the 48th day or 17 February 2010, and a second maximum of 60 EB counts on the 46th day. After the earthquake, the EBs fell below 30 . So, the enhancement in EBs between the 46th and 55th days occurred with an EB average of 10 and a sigma of slightly above 9 counts. The Chilean earthquake had its maximum EB at 10 days before as a strong fluctuation of a superPoisson EB distribution. This anomaly in electron flux has also been reported by another analysis (Zhang et al., 2012), which identified three $\mathrm{CR}$ electron peaks on the same days in 2010. The latter authors argue hat EB peaks were triggered by VLF electric field perturbation at the same positions. Moreover, ULF and ELF electric field perturbations were also detected prior to this earthquake, even if they occurred later on 24 and 25 February (Zhang et al., 2011). The geomagnetic activity was highly varying during the days of the peaks, even if the Ap index was less than 20. This was verified by the Dst (disturbance storm time) index, downloaded from the website http://wdc.kugi.kyoto-u.ac.jp/dstdir/, which indicated disturbed magnetic periods along the days with electron bursts.

\subsection{Summary of earthquakes with $M \geq 7.0$}

In these six cases, the time differences between EBs and earthquakes were longer than those of previously published works, where it had only been a few hours (Sgrigna et al., 2005; Fidani et al., 2008). These longer time differences are in agreement with the latest DEMETER IDP findings, where increases in energetic electron fluxes were observed a few days before earthquakes (Anagnostopoulos et al., 2010a, b; Sidiropoulos et al., 2011). Based on this, we set out to verify by how many and how high EBs increased prior to strong earthquakes over the entire DEMETER IDP survey period from 2005 to 2010. Data on earthquakes with $M \geq 7.0$ were considered during the same period, while strong aftershocks
$(M \geq 7.0)$ occurring after the main shocks were not considered for the analyses. Earthquakes with latitudes greater than $40^{\circ}$ were also excluded from the analyses as our criteria to select EBs considered only low L-shell electrons that were confined to low latitudes. Furthermore, earthquakes having depths less than $100 \mathrm{~km}$ were chosen. A total of 45 seismic events were selected and are listed in Table 1, together with a total of 56 EB peaks, which were frequently observed at the same times at different energy bands.

From the six reported cases, a sole criterion was chosen at this time of the analysis for EBs, and these data are shown in Table 1. These data are slightly different from those in Fig. 6, where different coordinate ranges were used. The database EBs were selected to have differences in latitude $\leq 5^{\circ}$ compared to the earthquake latitude and the EB conjugate points. The EB numbers that resulted were between \pm 20 days from the earthquake date and were summed over an interval of $24 \mathrm{~h}$. The reported anomalies in the number of EBs can be described as the number of sigmas above the average EB number in order to reach the peak. These EB distributions were super-Poisson, and were different among each other compared to the ratio between the averages and the sigmas. Being so, to calculate the probability that the peaks were not random, an optimal function had to be used to approximate EB distributions. The generalised Poisson distribution (Consul and Shoukri, 1985) was shown to be a good approximation of EB distributions in this study over the considered time intervals. A discrete random variable is said to have a generalised Poisson distribution if its mass function is given by

$$
\begin{gathered}
P_{n}(\lambda, \theta)=\frac{\lambda(\lambda+n \theta)^{n-1}}{n !} \exp (-\lambda-n \theta), n=0,1,2 \ldots \\
P_{n}(\lambda, \theta)=0, n>m, \theta<0
\end{gathered}
$$

and zero otherwise, where $\lambda>0, \max (-1,-\lambda / m) \leq \theta<1$ and $m(\geq 4)$ is the largest positive integer for which $\lambda+$ $\theta m>0$ when $\theta$ is negative. This study produced only superPoisson distributions, so $\theta>0$ always. $\lambda$ and $\theta$ could be obtained from the expressions of the moments (Ambagaspitiya and Balakrishnan, 1994), with $\lambda=\sqrt{\bar{n}^{3} / \bar{\Delta} n^{2}}$ and $\theta=1-$ $\sqrt{\bar{n} / \bar{\Delta} n^{2}}$, where $\bar{n}$ is the EB average and $\bar{\Delta} n^{2}$ is the EB variance being both calculated only considering quiet solar days. If $N$ is the EB number corresponding to the peak observed near an earthquake, the probability that the EB peak will not be a generalised Poisson fluctuation is $P=\sum_{n=0}^{N-1} P_{n}(\lambda, \theta)$.

Earthquake and EB data from 2005 to 2010 are summarized in Table 1: earthquake occurrence date and time, latitude, longitude, depth, magnitude $M$, the energy bands (e1...e6) with anomalous EBs, the time difference $D$ of the EB anomaly with respect to the earthquake, the EB L-shell, the EB peak number $N$, the EB average, the square root of the EB variance and the peak probability $P$. All considered EB peaks were characterised by the probability that they were not generalised Poisson fluctuations greater than $96 \%$, while there was always a probability greater than $97 \%$ for 
Table 1. Information regarding strong earthquakes and their corresponding EB anomalies during the whole DEMETER IDP database period.

\begin{tabular}{|c|c|c|c|c|c|c|c|c|c|c|c|c|c|}
\hline $\begin{array}{l}\text { Date } \\
\text { (YYYYMMDD) }\end{array}$ & $\begin{array}{l}\text { Time } \\
\text { (hhmmss) }\end{array}$ & $\begin{array}{l}\text { Lat } \mathrm{N} \\
\left({ }^{\circ}\right)\end{array}$ & $\begin{array}{l}\text { Long E } \\
\left({ }^{\circ}\right)\end{array}$ & $\begin{array}{l}\text { Depth } \\
(\mathrm{km})\end{array}$ & $M$ & $\mathrm{Ch}$ & $\begin{array}{l}D \text { time } \\
\text { (days) }\end{array}$ & $D / N$ & $L$ & $N$ & $\bar{n}$ & $\sqrt{\bar{\Delta} n^{2}}$ & $P(\%)$ \\
\hline \multirow[t]{2}{*}{20050328} & $16: 09: 36$ & 2.09 & 97.11 & 30 & 8.6 & $\mathrm{e} 2$ & -6 & 1 & 1.1 & 20 & 6.73 & 4.27 & 98.8 \\
\hline & & & & & & e3 & -7 & & 1.1 & 21 & 7.00 & 4.33 & 99.0 \\
\hline 20050724 & $15: 42: 06$ & 7.92 & 92.19 & 16 & 7.2 & no & - & - & - & - & - & - & - \\
\hline 20050816 & $02: 46: 28$ & 38.28 & 142.04 & 36 & 7.2 & no & - & - & - & - & - & - & - \\
\hline 20050909 & $07: 26: 43$ & -4.54 & 153.47 & 90 & 7.6 & $\mathrm{e} 3$ & -1 & 01 & 1.1 & 28 & 11.53 & 6.16 & 98.1 \\
\hline 20051008 & 03:50:41 & 34.54 & 73.59 & 26 & 7.6 & no & - & - & - & - & - & - & - \\
\hline \multirow[t]{3}{*}{20051114} & $21: 38: 51$ & 38.11 & 144.9 & 11 & 7 & $\mathrm{e} 2$ & -9 & 01 & 1.4 & 29 & 7.50 & 7.11 & 98.1 \\
\hline & & & & & & e3 & & & 1.4 & 32 & 8.94 & 7.79 & 98.1 \\
\hline & & & & & & $\mathrm{e} 4$ & & & 1.4 & 26 & 5.87 & 6.25 & 98.3 \\
\hline \multirow[t]{5}{*}{20060222} & $22: 19: 07$ & -21.32 & 33.58 & 11 & 7 & $\mathrm{e} 2$ & $-3,-5$ & 1 & 1.15 & 81 & 15.77 & 16.03 & 99.2 \\
\hline & & & & & & $\mathrm{e} 3$ & & & 1.15 & 78 & 14.73 & 16.44 & 98.9 \\
\hline & & & & & & e4 & & & 1.1 & 82 & 13.75 & 16.71 & 99.0 \\
\hline & & & & & & e5 & & & 1.1 & 63 & 11.18 & 11.55 & 99.4 \\
\hline & & & & & & e6 & & & 1.1 & 53 & 8.04 & 10.46 & 99.1 \\
\hline 20060503 & $15: 26: 40$ & -20.19 & -174.12 & 55 & 8 & no & - & - & - & - & - & - & - \\
\hline \multirow{2}{*}{20060717} & $08: 19: 26$ & -9.28 & 107.42 & 20 & 7.7 & $\mathrm{e} 4$ & -1 & 1 & 1.25 & 59 & 22.71 & 13.41 & 98.0 \\
\hline & & & & & & e5 & & & 1.25 & 33 & 13.33 & 7.82 & 97.5 \\
\hline 20061226 & $12: 26: 21$ & 21.8 & 120.55 & 10 & 7.1 & no & - & - & - & - & - & - & - \\
\hline 20070121 & $11: 27: 45$ & 1.07 & 126.28 & 22 & 7.5 & $\mathrm{e} 4$ & $-1,-17$ & 0 & 1.1 & 19 & 4.85 & 4.25 & 98.7 \\
\hline 20070325 & 00:40:01 & -20.62 & 169.36 & 34 & 7.1 & no & - & - & - & - & - & - & - \\
\hline 20070401 & $20: 39: 59$ & -8.47 & 157.04 & 24 & 8.1 & $\mathrm{e} 3$ & -3 & 01 & 1.1 & 42 & 8.05 & 9.08 & 98.8 \\
\hline 20070815 & $23: 40: 58$ & -13.39 & -76.6 & 39 & 8 & e5 & -6 & 0 & 1.25 & 49 & 21.09 & 9.07 & 99.1 \\
\hline \multirow[t]{2}{*}{20070902} & 01:05:18 & -11.61 & 165.76 & 35 & 7.2 & $\mathrm{e} 2$ & -5 & 0 & 1.25 & 76 & 20.21 & 17.30 & 98.5 \\
\hline & & & & & & $\mathrm{e} 3$ & & & 1.25 & 33 & 7.71 & 7.85 & 98.3 \\
\hline 20070912 & $11: 10: 27$ & -4.44 & 101.37 & 34 & 8.5 & no & - & - & - & - & - & - & - \\
\hline 20071114 & $15: 40: 50$ & -22.25 & -69.89 & 40 & 7.7 & no & - & - & - & - & - & - & - \\
\hline 20080220 & 08:08:30 & 2.77 & 95.96 & 26 & 7.4 & $\mathrm{e} 4$ & -7 & 0 & 1.1 & 225 & 29.83 & 43.08 & 99.1 \\
\hline \multirow[t]{2}{*}{20080320} & $22: 32: 58$ & 35.49 & 81.47 & 10 & 7.2 & $\mathrm{e} 4$ & $-1,-4,-8$ & 1 & 1.3 & 37 & 11.01 & 9.53 & 97.6 \\
\hline & & & & & & e5 & -8 & & 1.35 & 28 & 5.86 & 5.89 & 99.0 \\
\hline 20080409 & $12: 46: 13$ & -20.07 & 168.89 & 33 & 7.3 & no & - & - & - & - & - & - & - \\
\hline \multirow[t]{2}{*}{20080512} & 06:28:02 & 31 & 103.32 & 19 & 7.9 & $\mathrm{e} 2$ & -6 & 0 & 1.3 & 69 & 27.96 & 14.42 & 98.5 \\
\hline & & & & & & e5 & -4 & 1 & 1.35 & 26 & 11.22 & 5.02 & 99.0 \\
\hline 20080719 & 02:39:29 & 37.55 & 142.21 & 22 & 7 & e5 & +10 & 1 & 1.3 & 16 & 4.58 & 3.67 & 98.5 \\
\hline 20080929 & $15: 19: 32$ & -29.76 & -177.68 & 36 & 7 & no & - & - & - & - & - & - & - \\
\hline \multirow[t]{2}{*}{20081116} & $17: 02: 33$ & 1.27 & 122.09 & 30 & 7.4 & e3 & $-12,-16$ & 1 & 1.1 & 19 & 7.27 & 4.64 & 97.4 \\
\hline & & & & & & $\mathrm{e} 4$ & $+16, \quad+18$ & & 1.1 & 6 & 2.25 & 1.62 & 96.1 \\
\hline \multirow[t]{2}{*}{20090103} & $19: 43: 51$ & -0.41 & 132.88 & 17 & 7.7 & $\mathrm{e} 2$ & -1 & 1 & 1.2 & 19 & 4.30 & 3.82 & 99.3 \\
\hline & & & & & & $\mathrm{e} 3$ & & & 1.1 & 18 & 5.91 & 4.65 & 97.3 \\
\hline \multirow[t]{2}{*}{20090211} & $17: 34: 50$ & 3.89 & 126.39 & 20 & 7.2 & $\mathrm{e} 2$ & $-10,-14$ & 1 & 1.05 & 62 & 9.55 & 9.85 & 99.7 \\
\hline & & & & & & $\mathrm{e} 4$ & & & 1.05 & 17 & 5.63 & 3.25 & 99.5 \\
\hline 20090218 & $21: 53: 45$ & -27.42 & -176.33 & 25 & 7 & no & - & - & - & - & - & - & - \\
\hline \multirow[t]{2}{*}{20090319} & $18: 17: 40$ & -23.04 & -174.66 & 31 & 7.6 & $\mathrm{e} 2$ & -18 & 1 & 1.4 & 33 & 9.21 & 6.59 & 99.2 \\
\hline & & & & & & e3 & +2 & & 1.45 & 21 & 7.87 & 3.50 & 99.8 \\
\hline 20090528 & $08: 24: 47$ & 16.73 & -86.22 & 19 & 7.3 & $\mathrm{e} 3$ & -1 & 1 & 1.1 & 27 & 11.81 & 5.29 & 98.8 \\
\hline & & & & & & $\mathrm{e} 4$ & & & 1.1 & 33 & 18.53 & 6.60 & 97.0 \\
\hline 20090810 & $19: 55: 38$ & 14.1 & 92.9 & 24 & 7.5 & $\mathrm{e} 2$ & -10 & 1 & 1.2 & 126 & 39.63 & 27.22 & 98.6 \\
\hline & & & & & & e3 & & & 1.05 & 133 & 24.19 & 20.26 & 99.8 \\
\hline & & & & & & $\mathrm{e} 4$ & & & 1.05 & 48 & 16.64 & 7.47 & 99.8 \\
\hline 20090902 & 07:55:01 & -7.78 & 107.3 & 46 & 7 & $\mathrm{e} 2$ & $-2,-8$ & 0 & 1.1 & 56 & 15.89 & 10.85 & 99.3 \\
\hline & & & & & & $\mathrm{e} 3$ & & & 1.1 & 48 & 10.95 & 8.58 & 99.6 \\
\hline 20090929 & $17: 48: 11$ & -15.49 & -172.1 & 18 & 8.1 & e3 & $-2, \quad-4, \quad-6$ & 0 & 1.25 & 37 & 12.85 & 8.58 & 98.1 \\
\hline & & & & & & $\mathrm{e} 4$ & -12 & & 1.25 & 5 & 0.70 & 1.02 & 99.2 \\
\hline 20090930 & 10:16:09 & -0.72 & 99.87 & 81 & 7.6 & $\mathrm{e} 4$ & 0 & 1 & 1.1 & 7 & 1.69 & 1.64 & 98.6 \\
\hline & & & & & & e5 & -10 & & 1.1 & 9 & 2.10 & 1.88 & 99.2 \\
\hline 20091007 & $22: 18: 51$ & -12.52 & 166.38 & 35 & 7.8 & $\mathrm{e} 2$ & $0,-17,-20$ & 1 & 1.1 & 11 & 1.34 & 2.13 & 99.2 \\
\hline & & & & & & e3 & -14 & & 1.15 & 18 & 2.00 & 2.92 & 99.6 \\
\hline 20100103 & $22: 36: 28$ & -8.8 & 157.35 & 25 & 7.1 & e5 & -1 & 1 & 1.1 & 5 & 1.21 & 1.17 & 98.7 \\
\hline 20100112 & $21: 53: 10$ & 18.44 & -72.57 & 13 & 7 & $\mathrm{e} 2$ & 0 & 1 & 1.25 & 36 & 18.71 & 5.49 & 99.6 \\
\hline 20100226 & $20: 31: 27$ & 25.93 & 128.43 & 25 & 7 & $\mathrm{e} 2$ & $0,-2,-9,-13$ & 1 & 1.25 & 85 & 17.98 & 18.20 & 98.8 \\
\hline & & & & & & $\mathrm{e} 3$ & $+1, \quad+10$ & & 1.15 & 28 & 7.81 & 4.33 & 99.9 \\
\hline & & & & & & $\mathrm{e} 4$ & & & 1.15 & 16 & 7.90 & 3.53 & 97.1 \\
\hline & & & & & & e5 & & & 1.15 & 17 & 8.39 & 3.21 & 98.7 \\
\hline
\end{tabular}


Table 1. Continued.

\begin{tabular}{|c|c|c|c|c|c|c|c|c|c|c|c|c|c|}
\hline $\begin{array}{l}\text { Date } \\
\text { (YYYYMMDD) }\end{array}$ & $\begin{array}{l}\text { Time } \\
\text { (hhmmss) }\end{array}$ & $\begin{array}{l}\text { Lat } \mathrm{N} \\
\left({ }^{\circ}\right)\end{array}$ & $\begin{array}{l}\text { Long E } \\
\left({ }^{\circ}\right)\end{array}$ & $\begin{array}{l}\text { Depth } \\
(\mathrm{km})\end{array}$ & $M$ & $\mathrm{Ch}$ & $\begin{array}{l}D \text { time } \\
\text { (days) }\end{array}$ & $D / N$ & $L$ & $N$ & $\bar{n}$ & $\sqrt{\bar{\Delta} n^{2}}$ & $P(\%)$ \\
\hline 20100227 & $06: 34: 12$ & -36.12 & -72.9 & 22 & 8.8 & no & - & - & - & - & - & - & - \\
\hline \multirow[t]{2}{*}{20100404} & $22: 40: 43$ & 32.3 & -115.28 & 4 & 7.2 & $\mathrm{e} 2$ & $0,-2,-16$ & 01 & 1.35 & 31 & 8.23 & 7.94 & 97.8 \\
\hline & & & & & & $\mathrm{e} 5$ & +10 & 0 & 1.25 & 10 & 2.78 & 2.10 & 99.2 \\
\hline 20100406 & $22: 15: 02$ & 2.38 & 97.05 & 31 & 7.8 & e5 & -6 & 1 & 1.05 & 7 & 2.39 & 1.67 & 98.1 \\
\hline \multirow{4}{*}{20100509} & $05: 59: 42$ & 3.75 & 96.02 & 38 & 7.2 & $\mathrm{e} 2$ & $-4,-19,-20$ & 1 & 1.05 & 29 & 4.38 & 4.45 & 99.8 \\
\hline & & & & & & e3 & & & 1.0 & 19 & 6.99 & 4.99 & 96.8 \\
\hline & & & & & & $\mathrm{e} 4$ & & & 1.05 & 12 & 4.38 & 3.09 & 97.0 \\
\hline & & & & & & e5 & & & 1.05 & 12 & 3.53 & 2.74 & 98.6 \\
\hline 20100527 & $17: 14: 47$ & -13.7 & 166.64 & 31 & 7.1 & no & - & - & - & - & - & - & - \\
\hline 20100612 & $19: 26: 50$ & 7.88 & 91.94 & 35 & 7.5 & no & - & - & - & - & - & - & - \\
\hline 20100616 & $03: 16: 28$ & -2.17 & 136.54 & 18 & 7 & no & - & - & - & - & - & - & - \\
\hline 20100718 & $13: 34: 59$ & -5.93 & 150.59 & 35 & 7.3 & no & - & - & - & - & - & - & - \\
\hline
\end{tabular}

at least one EB peak to be detected before the earthquake. Local times are listed in Table 1 with the sub-orbit number, 0 -down orbit in local daytime (LD) and 1-up orbit in local nighttime (LN), indicating the local magnetosphere sun illumination or shadow at the time and position of EB increases.

There were 16 strong earthquakes out of a total of 45 with no anomalies in the number of EBs observed near seismic events during quiet solar days, and anomalies observed during days with $\mathrm{Ap}>20$ and Dst $<-30 \mathrm{nT}$ were discarded. Most of these 16 cases took place during the northern summer (May-September) when EB peaks have a maximum. For this, it is also more difficult to distinguish EB anomalies from EB fluctuations. The 29 earthquakes associated with EB peaks occurred mostly during the remaining months. Moreover, EB anomalies were observed less than 2 days before 11 earthquakes, and 7 earthquakes had anomalies occurring more than 10 days before. A total of 48 anomalies preceded earthquakes, with more than one EB peak associated with them, while only $5 \mathrm{~EB}$ anomalies followed earthquakes. Multiple EB peaks reached the maximum number of 5 in connection with one earthquake, and so they had to be considered relative to all energy bands when reported on the same line in Table 1. EB peaks appearing at different energy channels, but on the same day, were considered as contributors to this single anomaly. These EB peaks were relative to different energy bands when reported on different lines in Table 1, which corresponded to the indicated energy bands. EB peaks were observed in more than one energy channel corresponding to 20 earthquakes and up to five channels from e 2 to e 6 bands simultaneously. Some sudden EB increases were present without seismic activity.

Table 1 also reports that the $\mathrm{LN}$ frequency was greater than $\mathrm{LD}$, and $\mathrm{LN}$ covered all the time differences, even if the EB anomalies were more frequently detected near the earthquake time when EBs belonged to both $\mathrm{LN}$ and LD sub-orbits. The probability that EB fluctuations were not generalised Poisson flucuations was greater when EBs belonged to both $\mathrm{LN}$ and $\mathrm{LD}$ with respect to detections occurring along only $\mathrm{LN}$ or LD sub-orbits. Earthquake latitudes and longitudes resulted in not being influential for time differences, even though the greater number of seismic events, which were preceded by EB anomalies, occurred at longitudes $80^{\circ}-180^{\circ}$ in the Sumatra and Philippines regions (see Fig. 7a). From Fig. 7b it can be observed that EBs occurred up to 20 days before the earthquakes at lower L-shells. Furthermore, the time differences were generally larger with greater earthquake depth limits, as shown in Fig. 7c. Finally, the probability that EB fluctuations were not generalised Poisson fluctuations increased with greater magnitudes: $>98 \%$ for $M \geq 8$ earthquakes (see Fig. 7d).

\section{Conclusions}

A criterion to select EBs having a probability greater than $99 \%$ not to be Poisson fluctuations has been applied on high energy electron flux data at $70 \mathrm{keV}-2.34 \mathrm{MeV}$ recorded by IDP on DEMETER. Unlike past works, the L-shell parameter was not used here to compare EBs and earthquakes even if it was taken into account for the criteria selection. Time differences between these phenomena were considered within their limited latitudes, at the same time they had limited differences in latitude of $\pm 5^{\circ}$ between them. Only strong and shallow earthquakes were included in this study. The results were as follows:

1. Due to the full moons, the satellite attitude was changed, and then the values of the pitch angle were uncommon from October 2004 to March 2009. So, it can be concluded that this EB selection study is reliable for analysing IDP data and verifying the attitudes of the satellite reported in the attitude files.

2. The EB number followed seasonal variations at energy bands lower than $440 \mathrm{keV}$, with peaks occurring in the summer and winter, while EB valleys occurred in the spring and autumn. A comparison with the electron fluxes induced by lightning activity over the United States (Gemelos et al., 2009) showed the principal difference in having a unique maximum in the summer. To explain this discrepancy, it should be highlighted that 
a)

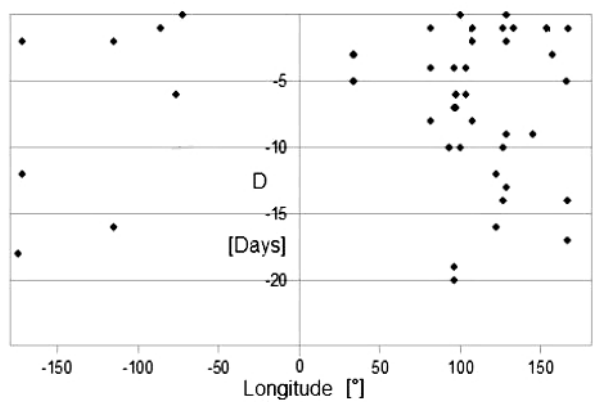

c)

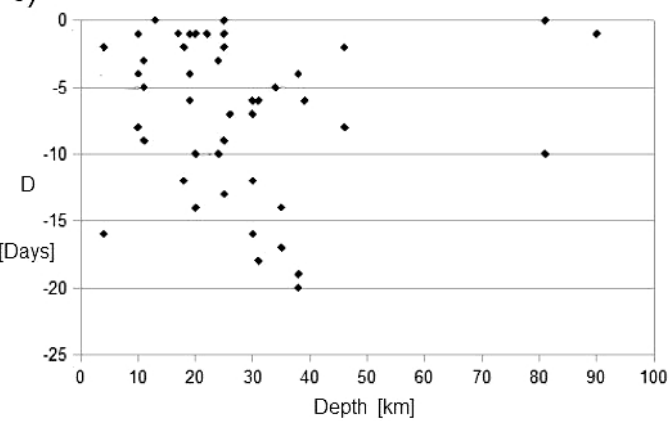

b)

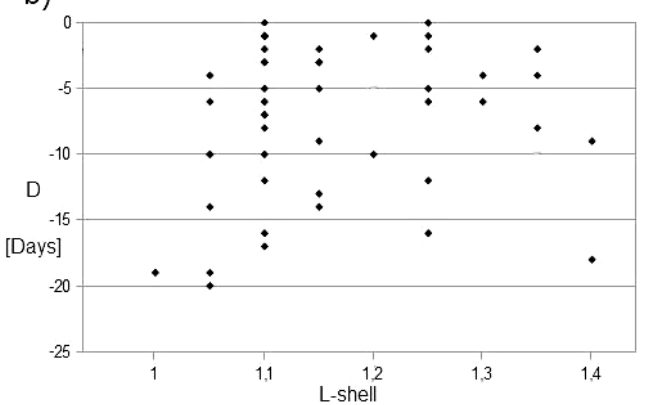

d)

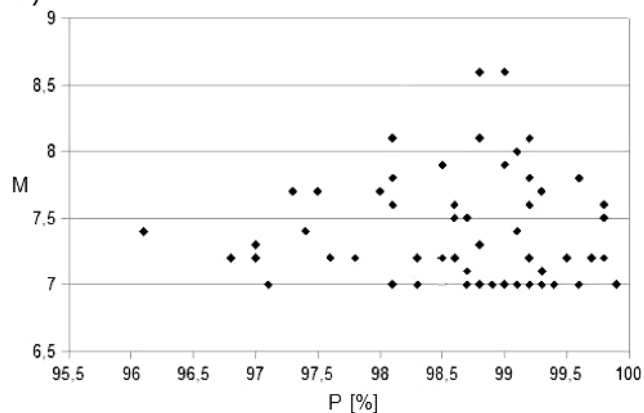

Fig. 7. Four graphs extracted from Table 1 by using only the events where EBs preceded Eqs: (a) the unequal earthquake longitude distribution; (b) distribution of EBs in L-shell and difference time $D, D$ increased with lower $L$; (c) a greater minimum depth for earthquakes occurrs at larger time difference with EBs occurrence; (d) EB anomalies with a probability greater than $96 \%$ for $M \geq 7$ earthquakes and with probability greater than $98 \%$ for $M \geq 8$ earthquakes.

the EBs studied in this work came from a completely different analysis on electron CRs, which was based on a relative intensity fluctuation of electron flux compared to the average of the period. Being so, high electron fluxes did not always mean high EB numbers. Furthermore, latitudes and L-shell coordinates of electrons were also different compared to previous studies. EB peaks were exclusively selected from the inner VAB, with $L<1.6$ and latitudes less than $20^{\circ}$, whereas the others were taken from the external VAB. The semiannual modulation of EB reflects the cross section variation of the geomagnetic field compared to solar wind (Vassiliadis et al., 2002), thereby still indicating the presence of a significant solar influence on EB activity. Here, one can conclude that a deeper and extended analysis should be conducted to better establish the threshold in geomagnetic parameters so to exclude solar influence, even if a Dst $<-30 \mathrm{nT}$ condition was used here to improve the EB selection.

3. The generalised Poisson probability that EB numbers are not statistical fluctuations is greater than $96 \%$ for seismic events with $M \geq 7.0$. This increased above $98 \%$ for $M \geq 8.0$ earthquakes. Each earthquake was preceded by EBs, of which at least one EB had a probability greater than $97 \%$ of not being a generalised Poisson fluctuation.
4. EB anomalies occurred for the most part at lower energy bands from e2 to e5, even if EB increases could not be observed at all of the e2-e5 bands. EB anomalies were more frequently detected before strong earthquakes and belonged to LN sub-orbits. However, short time differences between EB anomalies and earthquakes, as well as low EB probability, were preferred when EBs were detected along both $\mathrm{LN}$ and LD sub-orbits. The greater number of EB anomalies occurred in relation to earthquakes with longitudes between $80^{\circ}$ to $180^{\circ}$. This was not considered a genuine effect as it reflected the concentration of strong seismic events in the Sumatra and Philippine regions.

5. The maximum time difference $D$ (Fig. 7) between EBs and earthquakes was much larger for low L-shell compared to high L-shell. This suggest that, if it is due to a physical perturbation and if it starts during the earthquake preparation stage, the data do not contradict a perturbation, which moves upward crossing higher L-shells at later times. Figure 7c shows a trend for time difference $D$ to occur for earthquakes with greater minimum depth. 
Acknowledgements. The authors would like to thank the DEMETER Data Center for providing the IDP data and especially Dr. Sauvaud for the factor transformation of the IDP flux data into $\mathrm{CR}$ data. This paper was funded in part by the International Cooperation Project (2009DFA21480). Xuemin Zhang would like to thank the Chinese Scholarship Council and CEA for supporting this study as well as the INFN of Italy. Cristiano Fidani would like to thank the "Consulta delle Fondazioni delle Casse di Risparmio Umbre" for their financial support.

Edited by: N. Kerle

Reviewed by: K. Kudela and one anonymous referee

\section{References}

Abel, B. and Thorne, R. M.: Electron scattering loss in Earth's inner magnetosphere 1. Dominant physical processes, J. Geophys. Res., 103, 2385-2396, 1998.

Aleksandrin, S. Yu., Galper, A. M., Grishantzeva, L. A., Koldashov, S. V., Maslennikov, L. V., Murashov, A. M., Picozza, P., Sgrigna, V., and Voronov, S. A.: High-energy charged particle bursts in the near-Earth space as earthquake precursors, Ann. Geophys., 21, 597-602, doi:10.5194/angeo-21-597-2003, 2003.

Ambagaspitiya, R. S. and Balakrishnan, N.: On the compound generalized Poisson distribution, Astin Bull., 24, 255-263, 1994.

Anagnostopoulos, G., Rigas, V., Athanasiou, M., Iliopoulos, A., Vassiliadis, E., and Iossifidis, N.: Temporal Evolution of Energetic Electron Precipitation as a promising tool for Earthquake Prediction Research: Analysis of IDP/DEMETER Observations, in: Advances in Hellenic Astronomy during the IYA09, ASP Conference Series, edited by: Tsinganos K., Hatzidimitriou D., and Matsakos T., 424, 67-74, 2010a.

Anagnostopoulos, G., Rigas, V., and Vassiliadis, E.: Radiation belt electron precipitation in the upper ionosphere at middle latitudes before strong earthquakes, arXiv:1012.3588, 2010b.

Baker, D. N., Mason, G. M., Figueroa, Q., Colon, G., Watzin, J. G., and Aleman, R. M.: An overview of the solar, anomalous, and magnetospheric particle explore (SAMPEX) mission, Trans. Geosci. Remote Sens., 31, 531-541, 1993.

Boskova, J., Smilauer, J., Triska, P., and Kudela, K.: Anomalous behavior of plasma parameters as observed by Intercosmos 24 satellites prior to the iranian earthquake of 20 June 1990, Studia Geophys. Geod., 38, 213-220, 1994.

Brun, R.: ROOT An Object Orientable Data Analysis Framework User Guide 5.26, 470 pp., December 2009.

Buzzi, A.: DEMETER Satellite Data Analysis of SeismoElectromagnetic Signals, Doctoral Thesis, Univerity of Rome Tre, XIX Cycle, available at: http://lpce.cnrs-orleans.fr/www_ labo/labo_theses.php, last access: 3 April 2008.

Consul, P. C. and Shoukri, M. M.: The generalized Poisson distribution when the sample mean is larger than the sample variance, Comm. Stat. Sim. Comput., 14, 1533-1547, 1985.

Cotts, B. R. T., Inan, U. S., and Lehtinen, N. G.: Longitudinal dependence of lightning-induced electron precipitation, J. Geophys. Res., 116, A10206, doi:10.1029/2011JA016581, 2011.

Couet, O. and Goossens, M.: HBOOK Statistical Analysis and Histogramming Reference Manual, Information Technology Division, CERN Geneva, Switzerland, 193 pp., 1998.
Datlowe, D. W.: Differences between transmitter precipitation peaks and storm injection peaks in low altitude energetic electron spectra, J. Geophys. Res., 111, A12202, doi:10.1029/2006JA011957, 2006.

Datlowe, D. W., Imhof, W. L., Gaines, E. E, and Voss, H. D.: Multiple Peaks in the Spectrum of Inner Belt Electrons, J. Geophys. Res., 90, 8333-8342, 1985.

Fidani, C. and Battiston, R.: Analysis of NOAA particle data and correlations to seismic activity, Nat. Hazards Earth Syst. Sci., 8, 1277-1291, doi:10.5194/nhess-8-1277-2008, 2008.

Fidani, C., Battiston, R., and Burger, W. J.: A study of the correlation between earthquakes and NOAA satellite energetic particle bursts, Remote Sens., 2, 2170-2184, 2010.

Galper, A. M., Koldashov, S. V., Murashov, A. M., Maslennikov, L. V., Shvetz, N. I., and Voronov, S. A.: High energy charged particle waves on the low boundary of the radiation belt, Proc. of 25th ICRC, 2, 349-350, 1997.

Gemelos, E. S., Inan, U. S., Walt, M., Parrot, M., and Sauvaud, J. A.: Seasonal dependence of energetic electron precipitation: Evidence for a global role of lightning, Geophys. Res. Lett., 36, L21107, doi:10.1029/2009GL040396, 2009.

Grigoryan, O., Kudela, K., Rothkaehl, H., and Sheveleva, V.: The electron formations under the radiation belts at L-shells 1.2-1.9, Adv. Space Res., 41, 81-85, 2008.

Imhof, W. L., Gaines, E. E., and Reagan, J. B.: High-Resolution Spectral Features Observed in the Inner Radiation Belt Trapped Electron Population, J. Geophys. Res., 86, 2341-2347, 1981a.

Imhof, W. L., Anderson, R. R., Reagan, J. B., and Gaines, E. E: The Significance of VLF Transmitters in the Precipitation of Inner Belt Electrons, J. Geophys. Res., 86, 11225-11234, 1981b.

Kanekal, S. G., Baker, D. N., and McPherron, R. L.: On the seasonal dependence of relativistic electron fluxes, Ann. Geophys. 28, 1101-1106, doi:10.5194/angeo-28-1101-2010, 2010.

Krunglanski, M.: UNILIB Reference Manual, Belgisch Institute Voor Ruimte-Aeronomie, 2002.

McIlwain, C. E.: Magnetic Coordinates, Space Sci. Rev., 5, 585598, 1966.

Millan, R. M. and Thorne, R. M.: Review of radiation belt relativistic electron losses, J. Atmos Sol.-Terr. Phys., 69, 362-377, 2007.

Molchanov, O. A. and Majaeva, O. A.: Statistic features of ULF and VLF electromagnetic emissions in upper ionosphere and magnetosphere above centers of earthquakes, Cosmic Res., 32, 150157, 1994.

Molchanov, O. A., Mazhaeva, O. A., Golyavin, A. N., and Hayakawa, M.: Observation by the Intercosmos-24 satellite of ELF-VLF electromagnetic emissions associated with earthquakes, Ann. Geophys., 11, 431-440, 1993, http://www.ann-geophys.net/11/431/1993/.

Parrot, M., Berthelier, J. J., Lebreton, J. P., Sauvaud, J. A., Santolík, O., and Blecki, J.: Examples of unusual ionospheric observations made by the DEMETER satellite over seismic regions, Phys. Chem. Earth, 31, 486-495, 2006.

Pulinets, S. and Boyarchuk, K.: Ionospheric Precursors of Earthquakes, Springer, ISBN3-540-20839-9, 2004.

Sauvaud, J. A., Moreau, T., Maggiolo, R., Treilhou, J.-P., Jacquey, C., Cros, A., Coutelier, J., Rouzaud, J., Penou, E., and Gangloff, M.: High-energy electron detection onboard DEMETER: The IDP spectrometer, description and first results on the inner belt, Planet Space Sci., 54, 502-511, 2006. 
Sauvaud, J. A., Maggionlo, R., jacquey, C., Parrot, M., Berthelier, J.-J., Gamble, R. J., Craig, J.-J., and Rodger, J.: Radiation belt electron precipitation due to VLF transmitters: satellite observations. Geophys. Res. Lett., 35, L09101, doi:10.1029/2008GL033194, 2008.

Sgrigna, V., Carota, L., Conti, L., Corsi, M., Galper, A. M., Koldashov, S. V., Murashov, A. M., Picozza, P., Scrimaglio, R., and Stagni, L.: Correlations between earthquakes and anomalous particle bursts from SAMPEX/PET satellite observations, J. Atmos. Sol.-Terr. Phys., 67, 1448-1462, 2005.

Sgrigna, V., Buzzi, A., Conti, L., Picozza, P., Stagni, C., and Zilpimiani, D.: Seismo-induced effects in the near-earth space: Combined ground and space investigations as a contribution to earthquake prediction, Tectonophys., 431, 153-171, 2007.

Sidiropoulos, N. F., Anagnostopoulos, G., and Rigas, V.: Comparative study on earthquake and ground based transmitter induced radiation belt electron precipitation at middle latitudes, Nat. Hazards Earth Syst. Sci., 11, 1901-1913, doi:10.5194/nhess-111901-2011, 2011.

Vassiliadis, D., Klimas, A. J., Kanekal, S. G., Baker, D. N., and Weigel, R. S.: Long-term-average, solar cycle, and seasonal response of magnetospheric energetic electrons to the solar wind speed, J. Geophys. Res., 107, 1383-1400, 2002.
Voronov, S. A., Galper, A. M., Koldashov, S. V., Kirillov-Ugriumov, V. G., Michailov, V. V., Popov, A. V., and Chesnokov, V. Yu.: Registration of Sporadic Increase of High Energy Particle Flux near Brazilian Anomaly Region, Proc. of 20th ICRC, 4, 451452, 1987.

Zhang, X., Liu, J., Shen, X., Parrot ,M., Qian, J., Ouyang, X., Zhao, S., and Huang, J.: Ionospheric perturbations associated with the M8.6 Sumatra earthquake on 28 March 2005, Chinese J. Geophys., 53, 567-575, 2010.

Zhang, X., Zeren, Z., Parrot, M., Battiston, R., Qian, J., and Shen, X.: ULF/ELF ionospheric electric field and plasma perturbations related to Chile earthquakes, Adv. Space Sci., 47, 991-1000, 2011.

Zhang, Z., Li, X., Wu, S., Ma, Y., Shen, X., Chen, H., Wang, P., You, X., and Yuan, Y.: DEMETER satellite observations of energetic particle prior to Chile earthquake, Chinese J. Geophys., 5, 15811590, English version at: arXiv:1011.3592v1, 2012. 